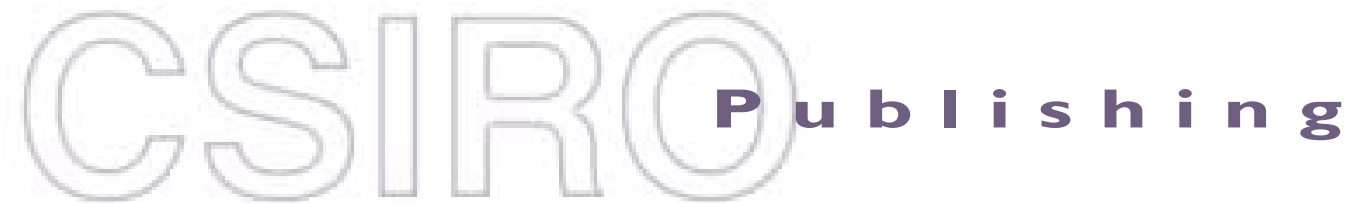

\section{Publications of the Astronomical Society of Australia}

Volume 19, 2002

(C) Astronomical Society of Australia 2002

An international journal of astronomy and astrophysics

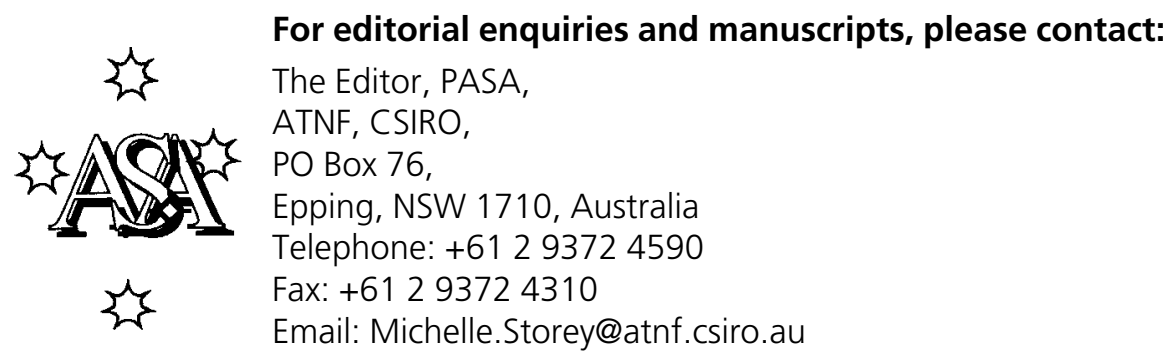

For general enquiries and subscriptions, please contact: CSIRO Publishing PO Box 1139 (150 Oxford St)

Collingwood, Vic. 3066, Australia

Telephone: +6139662 7666

Fax: +61 396627555

Email: publishing.pasa@csiro.au

C S I RO

PUBLISHING Published by CSIRO Publishing

for the Astronomical Society of Australia

www.publish.csiro.au/journals/pasa 


\title{
The Use of Online Discussion Forums as a Learning and Teaching Tool in Astronomy
}

\author{
Margaret Mazzolini \\ School of Biophysical Sciences and Electrical Engineering, \\ Mail Box 31, Swinburne University of Technology, \\ PO Box 214, Hawthorn, Vic. 3122, Australia \\ mmazzolini@swin.edu.au
}

Received 2002 June 10, accepted 2002 August 16

\begin{abstract}
Swinburne Astronomy Online (SAO) is an online graduate program in astronomy intended particularly for amateur astronomers, science communicators and educators. SAO emphasises the development of conceptual understanding and good communication skills in astronomy. The use of asynchronous discussion forums is a central feature of SAO. These 'online tutorials' were introduced partly with the intention of breaking down the isolation of distance education, but mainly to provide a venue where students could deepen their conceptual understanding of astronomy and hone their science communication skills while discussing course material with other students and instructors.

In this project aspects of the use of discussion forums in SAO were investigated, with comparisons made between average marks awarded for discussion forum contributions and those awarded for other assessment tasks, plus analysis of the rate at which students post to the forums, and an investigation of whether gender makes a significant difference to participation rate and assessment results. Responses by students to surveys that probe their ability to express themselves and communicate with classmates in this online form of distance education are discussed. Finally, possible implications for the use of discussion forums as a supplement to face-to-face teaching in astronomy are explored.
\end{abstract}

Keywords: astronomy education

\section{Overview}

Swinburne Astronomy Online (SAO), an online suite of programs in Master/Graduate Diploma/Graduate Certificate of Science in Astronomy, currently teaches just over 230 students located in approximately 34 countries. With 15 units (subjects) available in all, SAO is designed and promoted with the intention of teaching people about astronomy, rather than producing professional research astronomers.

More general information about the SAO program and its assessment mix can be found in Mazzolini (2000), Germany \& Mazzolini (2002), and at the SAO website. ${ }^{1}$ This paper will focus on the use of asynchronous discussion forums as a tool to aid learning and teaching in online astronomy courses.

\section{Desired Learning Outcomes}

The use of asynchronous discussion forums is a key feature of the SAO program and its assessment mix. Desired learning outcomes for SAO units typically include the ability to

- identify and correct common misconceptions,

- demonstrate knowledge and understanding of course content,

- discuss basic principles and concepts in a non-technical way understandable to the wider public,

- be able to research a topic in astronomy in detail,

${ }^{1}$ http://www.astronomy.swin.edu.au/sao/
- develop an increased perception of the limits of our understanding of astronomy, and

- develop an increased awareness of social and scientific implications of astronomy.

As can be seen from this list, the development of conceptual understanding of astronomy, plus good communication skills, are emphasised in SAO. These learning outcomes are particularly relevant to the discussion forum component of the program. They are chosen with the composition of the SAO student body in mind - SAO students are predominantly amateur astronomers who often give occasional presentations on astronomy in their astronomy associations and local schools, plus current and would-be science educators, communicators, and journalists.

\section{Discussion Forum Contributions}

A central requirement of the design of $\mathrm{SAO}$ was to provide a way by which students could deepen their conceptual understanding of astronomy by means of student-student and student-instructor discussions, despite its distance education format.

SAO students communicate with each other and with their instructors through online 'asynchronous' discussion forums, making forum postings at times to suit themselves. Students are allocated to discussion forum groups consisting of a maximum of approximately 30 students, each under the guidance of an instructor who is a professional astronomer. 
The discussion forum component of $\mathrm{SAO}$ was designed to encourage active learning (Mazzolini 2000) as a key component of online learning (Paloff \& Pratt 1999). A new discussion forum is opened up every two weeks for each student group. Students are required to post questions and/or extension comments about the current course material, and to attempt to answer each other's questions. Instructors act as 'guides on the side', aiding the discussions by contributing extra information and follow-up questions. They intervene in discussion threads that have 'gone off the rails', but avoid dominating the discussions. In this approach it is vital that the students see each other as a resource to obtain answers to their questions, rather than rely on the instructors as oracles. Our research has indicated that if instructors take too high a profile in discussions, they can tend to limit student participation and cut short the length of the discussions (Mazzolini \& Maddison 2002).

SAO students are informed that, at the end of the teaching semester, they will be asked to nominate which of their discussion forum postings are to be assessed. The intention is to motivate students to strive continually to make better postings, in order to have a good range to choose from at the semester's end. Counting forum contributions towards the final assessment grades recognises the time and effort students put in to researching and composing their forum contributions, and encourages contributions from all, not just the most confident (Mazzolini 2000). The discussion forum component of the final grade is made up of marks awarded for the nominated forum contributions, plus a relatively small 'participation mark' component intended to reward regular participation.

Relevant astronomy press releases can be channelled into the discussion forums. Instructors cannot only continue to teach while travelling but can also make postings about the progress (or otherwise) of observing trips, or 'hot' topics being discussed at conferences they may be attending at the time.

\section{Why Asynchronous?}

The discussion forums operate in asynchronous mode, where students make postings at times to suit themselves, rather than in synchronous mode, where the students and instructor would all be logged in and communicating in real time. Discussions conducted via asynchronous discussion forums may sound like a clumsy way to interact, but they can be surprisingly flexible and engaging, providing a form of peer group contact so frequently missing in distance education and also encouraging deep learning.

Necessity was the initial motivation for the use of asynchronous forums, as SAO students are located in most of the world's time zones and tend to be busy people, many with work commitments that require them to travel frequently. SAO instructors are also spread around the world, and so synchronous communication sessions are out of the question. Instead, in SAO, conversation threads build up on the forums as geographically separated students come online in their particular time zones. Relationships are established between students who have never met or visited each other's countries but who share a passion for astronomy.

In SAO it has become evident that asynchronous discussion forums have advantages other than those dictated by time zones and busy schedules. Asynchronous discussions give students (and instructors) time to research and reflect upon current discussion topics before they post answers to each other's questions. SAO students are taught when researching and composing their postings to use Internet resources such as web searches and an online encyclopedia as well as textbooks.

It is likely that the use of asynchronous discussions also encourages non-native English speakers to take part in online forums, because it allows them sufficient time to compose contributions, then post their replies when ready. Participation in synchronous 'chat room' type discussions can be very difficult for slow typists, but much more so for non-native English speakers. In online synchronous chat room discussions involving more than a few participants, it is easy for the various discussion 'threads' (topics) to become hopelessly entangled. In contrast, in asynchronous forum discussions, each discussion thread is clearly organised under its own heading, which is an extremely important consideration in a forum with thirty or so active contributors.

In each semester, there are six consecutive, two-week discussion forums for each unit, and students are required to contribute in each discussion forum. In the next section the rate at which students participate in practice is investigated.

\section{Analysis}

As outlined above, SAO participants are required to make at least two postings to the subject discussion forums in every two-week period. At least one of those postings should be a question or comment about the course content for that two-week period, and at least one other an answer to another participant's query. At the end of each semester, the participants themselves nominate which of their forum contributions should be assessed. They also receive a component of their overall mark for 'regular participation'.

When introducing any new component into the assessment mix for a course, it is important to see how its results compare to those from the other, more traditional assessment components. In this study the average marks given to SAO students for discussion forum contributions are compared to those given for the other assessment components.

Although students in SAO are only required to make a minimum of two forum postings in every two-week period, many post significantly more often than that. One of the aims of this study was to determine how often SAO students $d o$ post on average, and in particular, if there is a significant difference between the rate at which male as 
Table 1. Average marks (plus standard deviations shown in brackets) for SAO assessment tasks over two semesters

\begin{tabular}{llllll}
\hline & \multicolumn{5}{c}{ Average marks (with standard deviations) } \\
\cline { 2 - 6 } & CMTs & Essays & Projects & $\begin{array}{l}\text { Discussion } \\
\text { forums }\end{array}$ & Total marks \\
\hline Semester 2, 2000 & $87(11)$ & $75(16)$ & $79(15)$ & $79(13)$ & $79(11)$ \\
Semester 1, 2001 & $88(14)$ & $75(18)$ & $74(21)$ & $74(19)$ & $76(18)$ \\
Over both semesters & $88(14)$ & $75(17)$ & $75(19)$ & $75(19)$ & $77(14)$ \\
\hline
\end{tabular}

compared to female students post to forums. SAO has a male-dominated student population, averaging $81 \%$ males over the two semesters studied in this project. Does being a female student in a predominantly male group act as a disincentive when it comes to making postings online?

Each semester, SAO students respond to independent survey questions by Swinburne's Quality Education Unit. Their responses to questions relating to their opportunity to express themselves and communicate with their fellow students are analysed here. As communications in SAO are conducted almost entirely through discussion forums, answers to these questions can be expected to relate directly to their experience with forum discussions.

\subsection{Comparing Performance for Different Modes of Assessment}

SAO students receive marks for four types of assessment tasks: computer-managed tests (CMTs), essays, projects, and discussion forum contributions. The CMTs are openbook, multiple choice tests designed to reward students for studying the broad range of course material covered by each unit. The essays are intended to develop students' science communication skills at researching and synthesising relevant information, and at presenting it clearly and concisely for the non-specialist reader. The projects build on those communication skills, but also are intended to develop skills in researching and critically analysing a particular topic in depth. Finally, as already discussed, students receive marks for their participation plus for selected contributions to discussion forums that act as asynchronous 'online tutorials'. In the forum discussions students clarify their own understanding of key concepts and further develop their science communication skills by answering each other's questions, often in considerable depth.

Average marks have been calculated for all four assessment components, plus overall total marks, for SAO students over two semesters ( 219 unit enrolments in semester 2,2000 , and 265 unit enrolments in semester 1,2001). This represented assessment results from students grouped into 15 discussion forums. (The results from a further three forums were excluded as complete breakdowns of marks were unavailable.) Table 1 shows the average marks (plus standard deviations shown in brackets) for SAO assessment tasks over the two semesters. Note that standard deviations are used here as indicators only, as the marks for all assessment tasks have skewed distributions rather than normally distributed ones.

The average marks awarded for forum discussions are quite consistent with those awarded for the other assessment tasks. This is encouraging from the point of view of validity of the forum component of the assessment.

Not surprisingly, the open-book CMTs receive the highest average marks and have the smallest standard deviations. The standard deviation for the discussion forums is quite large, which is desirable as it suggests that the discussion forums are good tools to discriminate between the most and least capable students. Comparable averages to the other forms of assessment, plus relatively large standard deviations, suggest that the grading of discussion forum participation in $\mathrm{SAO}$ forms a useful component of the overall assessment mix.

However it is also possible that the large standard deviation for discussion forums might indicate that the forum marks lack reliability. Table 1 shows the marks awarded for all assessment tasks, including discussion forums, for each semester. Over our admittedly small sample of two semesters, there is no significant discrepancy between the marks for any of the tasks, including the discussion forums, but it will be necessary to keep a watch on the average marks for more than two semesters before it can be claimed with any real confidence that the marking is reliable. Even consistent averages are not in themselves enough to indicate that instructors mark consistently. To be confident of that, average marks and standard deviations for similar groups should be compared, and coordinators need to monitor and compare selections of marked work - for example, by requiring instructors to swap and cross-mark their 'best' and 'worst' examples.

\subsection{Frequency of Posting to Discussion Forums}

As Table 2 shows, most SAO students make significantly more than the required minimum of two postings per twoweek period. Does this apply to both male and female students? As discussed above, one aim of this study was to see whether women in the predominantly male SAO student population had posted relatively less often to the discussion forums than had the male SAO students.

The average number of postings per forum for both women and men are shown in Table 2 for all units, and also for introductory as compared to advanced units. The average number of postings per discussion forum for women 
Table 2. Average numbers of postings per discussion forum (plus standard deviations shown in brackets) for males and females in SAO discussion forums over two semesters

\begin{tabular}{ll}
\hline & $\begin{array}{l}\text { Average number of postings } \\
\text { (with standard deviations) } \\
\text { per discussion forum* }\end{array}$ \\
\hline Females (all units) & $4.5(2.3)$ \\
Males (all units) & $3.9(1.2)$ \\
Females (introductory units) & $4.0(1.7)$ \\
Males (introductory units) & $3.5(1.0)$ \\
Females (advanced units) & $5.4(2.9)$ \\
Males (advanced units) & $4.7(1.2)$ \\
\hline
\end{tabular}

* Results are shown for all units, plus breakdowns for introductory units (which contain a high proportion of students new to SAO) and advanced units (where students have previously studied with SAO). These statistics are based on averages from 108 two-week discussion forum discussions with 484 student enrolments in all, 92 of which are by female students.

and men differ (4.5 for women and 3.9 for men). According to the most relevant statistical test (the Independent Samples T-Test), this difference is only slightly significant. This is complicated by the fact that, although SAO students study part-time, they do often study more than one unit at a time, and so the same student can be enrolled in more than one unit. (Typically the average is approx 1.5 unit enrolments per semester per student.) Because of this, the samples from different forums are in fact not entirely independent, and this weakens any claim of a statistically significant difference between posting rates. Therefore, to the accuracy of this study, women and men post equally often, on average, to SAO forums - certainly the evidence does not support the theory that women might post less often than men in these male-dominated forums. Further, both women and men post approximately twice as often as they are required to do so, indicating that these forums are a well-used component of the SAO program.

This conclusion is interesting, because anecdotal responses from at least some female SAO students suggest that they think that they post less often than the men, although that may be a response conditioned by the dominance of postings by male students in forums due purely to the gender imbalance $(80 \%$ male) rather than by any actual differences in posting rates.

According to one female SAO student, 'After $21 / 2$ years of newsgroup discussions, I think I have observed differences in the gender response. I can also give some prospective to my own. I suspect there might be a difference between younger women and older women here as well. It is my bias - not a statistical evaluation but a gut feeling - that women, at least of my vintage, are more uncomfortable making entries, and make fewer. In my own class rooms when I am teaching — face-to-face it seems clear that men answer questions more confidently (even when wrong) and more frequently... . The problem is particularly acute in a forum where women are a small minority. This seems to be true here at Swinburne. Most of my classes have had only 2 or 3 women. And I think
Table 3. Average marks awarded for discussion forum contributions (plus standard deviations shown in brackets) for female students as compared to all students in SAO forums over two semesters

\begin{tabular}{ll}
\hline Students (all units) & $\begin{array}{l}\text { Average percentage mark for } \\
\text { discussion forum contributions }\end{array}$ \\
\hline Females & $79(13)$ \\
Males & $71(22)$ \\
\hline
\end{tabular}

we tend to submit one question and one answer/week, as required. I certainly am not comfortable with doing it. But I grit my teeth and carry on. I don't know what you should do about all this.'

As suggested by this student, it is possible that female participation rates may vary with age of the participant, but this study does not include a large enough number of women participants to test that hypothesis.

The distinction between introductory and advanced units in SAO is not absolute. While almost all students new to SAO take at least one introductory unit in their first semester, in subsequent semesters students may take more introductory units alongside advanced ones. Despite that tendency to mix introductory and advanced level students, the average numbers of postings per forum for both female and male students groups (see Table 2) in units labelled as introductory, as compared to those labelled as advanced, are significantly different, according to the most appropriate statistical test (One-Way Analysis of Variance). The difference is marked, even though its significance is again weakened somewhat by the fact that the same students can be enrolled in more than one unit, and so our data does suggest that students in advanced units may post more often than do students in introductory units.

Table 3 shows the average marks for forum contributions, scored over two semesters by female students, as compared to the average marks scored by the whole student cohort. If it could be assumed that discussion forum marks are distributed normally, then a statistical analysis of these marks would indicate that female students score on average slightly higher than do the male students for forum contributions. However in practice, given the typically skewed distributions of these marks, it is hard to conclude that there is any significant difference, so, to the accuracy of this study, females and males score equally highly on average for discussion forum assessment.

It will be interesting to repeat this analysis in the future, when more semesters of data are available, to see if the women in SAO do in fact contribute to the forums more often and receive higher marks for their forum assessment on average.

\subsection{Student Survey Results}

One motivation for the use of discussion forums in online education is to try to break down the isolation commonly felt by distance education students. From an educational 


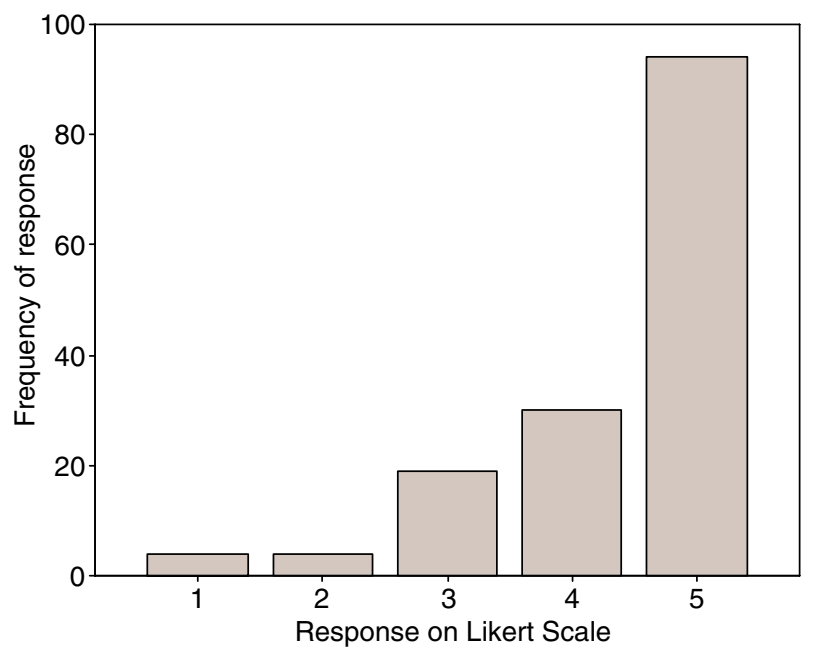

Figure 1 SAO student survey responses over two semesters on a 1-5 Likert Scale to the statement 'Encouragement was given to students to express themselves.' On the scale $1=$ strongly disagree, $2=$ disagree, $3=$ neither, $4=$ agree, $5=$ strongly agree; the average response was 4.4 out of 5 (standard deviation 1.0).

point of view, another key motivation is the desire to add a 'tutorial' element to distance education in order to improve conceptual understanding through student-student and student-instructor dialogue in forums. Certainly discussion forums work for many: to quote one SAO student, 'It's more like the classic Greek form of tuition, with the course tutors and students sitting round a forum discussing the subject. It's live tuition. Once again, better than a real class-bound course with lectures and tutorials.'

The Swinburne Quality Education Unit conducts anonymous surveys of SAO students each semester. Students are asked to respond on a 1-5 Likert Scale to various statements about $\mathrm{SAO}$, including two questions relating to student communication:

(A) 'Encouragement was given to students to express themselves.'

(B) 'I communicated more about course material with my classmates in this unit than I have with classmates in face-to-face instruction.'

Given that SAO discussions are structured around student contributions, so it would be reasonable to hope for a good response to statement (A), but the possibility of obtaining even a neutral response to statement (B) could be 'a big ask' for any form of distance education. Figures 1 and 2 show student responses to these statements over the twosemester period.

Figure 1 shows that statement (A) scored the extremely high average response of 4.4 out of a possible 5, indicating that SAO students do feel that the program encourages students to express themselves. This favourable response could be attributed in part to the essay and project assessment components as well as to discussion forum contributions. However it is reasonable to conclude that such a high overall rating indicates that students are comfortable with their ability to contribute to discussion forums.

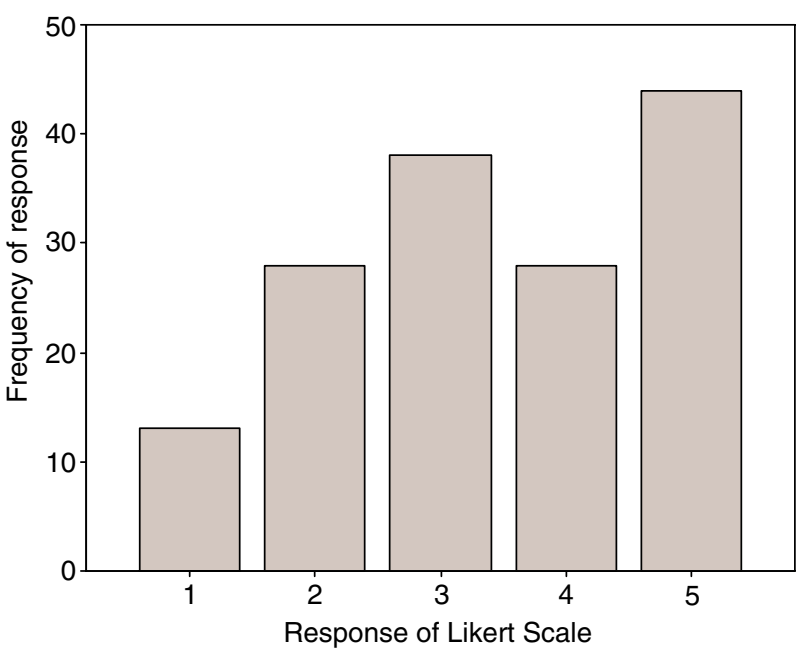

Figure 2 SAO student survey responses over two semesters on a 1-5 Likert Scale to the statement 'I communicated more about course material with my classmates in this unit than I have with classmates in face-to-face instruction.' On the scale $1=$ strongly disagree, $2=$ disagree, $3=$ neither, $4=$ agree, $5=$ strongly agree, the average response was 3.4 out of 5 (standard deviation 1.3).

Figure 2 shows that statement (B) scored a respectable 3.4 average response out of a possible 5. A neutral response, i.e. one averaging only 3.0 , would have indicated that SAO students felt that they communicated with each other in SAO as well as they have in previous faceto-face instruction, which would be an excellent result given the limitations of distance education. In fact, SAO exceeded expectations, scoring 3.4, indicating that, by a slight majority, students felt that they had communicated more with each other on average online than in previous face-to-face instruction.

\section{Issues Arising from the Use of Discussion Forums}

The need to rely primarily on asynchronous forums to communicate with students presents a new challenge to instructors used to the spontaneity of face-to-face teaching. University instructors tend to be good at 'just in time' course preparation and presentation as they juggle the many conflicting demands of university life. However online education requires advance planning — instructors need to plan the way they want the discussion forums to fit into their teaching programs, and adopt strategies for their success. Finding a balance between the extremes of leaving forums 'leaderless,' or of being overly interventionist and answering all the students' questions, requires a rethink of conventional teaching approaches, and instructors need to employ good time-management skills (Paloff \& Pratt 2001).

Another study (Mazzolini \& Maddison 2002) found that the rate at which instructors contribute to discussion forums influences the level of student participation with high levels of instructor participation correlating with lower than normal levels of student participation. Of course, instructors are not the only participants who may tend to dominate discussions. As in any face-to-face tutorial, one or two articulate and assertive students may 
dominate an online discussion if strategies are not developed to handle these situations. It helps to require all students to post contributions. However students who are tentative about Internet communications or who have a weak background knowledge of astronomy may still be daunted by frequent postings by other students who answer questions as soon as they are posted, and seemingly know everything about the subject material.

Among its largely 'mature age' students, SAO has more than its fair share of participants who are highly experienced amateur astronomers and science communicators. However the student group also includes others who are studying astronomy for the first time and may have even bought their first computer to do so. In SAO the terms 'black belters' and 'white belters' are used to refer to these two groups. This terminology has proven helpful as a humorous, non-pejorative way of referring to the participation of both beginners and experts in a forum discussion. For example, in a situation where enthusiastic 'expert' participants are tending to answer everyone's questions straight away in a two-week discussion forum, without leaving sufficient time for beginners to research and post answers, instructors can post reminders such as 'To all the black belters out there - you know who you are! - please just post questions this week and give us mere white belters a chance to answer them. Then you can polish off the remaining tricky questions next week.' Less confident students will tend to make light of their beginner status by identifying themselves in ways such as: 'I'm just a mere white belter but I think that the answer might be...'

At present, online courses available around the world tend to be in non-technological and non-mathematical discipline areas. Teaching mathematical material online has its own special challenges. The way in which HTML supports maths is clumsily at best, and, in particular, commercial online delivery systems tend not to support maths at all with their forum software. Although SAO is taught primarily from a conceptual rather than mathematical basis, maths is used where necessary and students (and instructors) often need to be able to include a small amount of maths in their discussion forum postings. Also, teaching astronomy online would be very difficult and limited if it were not possible to include astronomical images (and sketches) in postings.

In $\mathrm{SAO}$, the discussion forums are currently conducted using newsgroup (newsreader) technology. Students can use Netscape Communicator (preferred) or Outlook Express to view and make postings, and this technology supports the insertion of images inline. This also provides a reasonable fix for the problem of including mathematics in forum postings — the mathematics can be created and converted to jpeg image files using software such as Equation Editor (or MathType), then inserted inline as images into newsgroup postings. Of course, all such technological 'fixes' need good IT and helpdesk support for both students and staff, as does online education in general.

\section{Conclusion and Outlook}

SAO learning outcomes stress the development of conceptual understanding in astronomy, plus development of good communication skills. With those learning outcomes particularly in mind, online discussion forums are used in SAO to allow direct, although asynchronous, studentstudent and student-instructor communication despite the usual limitations of distance education. This study has investigated several aspects of the way discussion forums have been conducted and assessed in SAO over a two-semester period.

A study of how the marks were awarded as assessment for these forum discussions, as compared to the other components of the SAO assessment mix, found that they were comparable, and that male and female students receive on average the same marks for newsgroup participation. SAO students post to the forums on average significantly more often than they are required to, but to the accuracy of this study, there is no significant difference between the rate at which female and male students participate, even though women are heavily outnumbered in SAO. However students in 'advanced' SAO units do appear to post more often than do those in 'introductory' units, and, on average, all SAO students choose to post to forums significantly more often than they are required to do so.

SAO students are asked to respond to anonymous, independent survey questions each semester. Over the two semesters of this study, student responses to questions about their ability to express themselves and communicate with classmates were extremely positive, given that SAO is a form of distance education. SAO is an atypical online program in that the course material is not online - instead it is provided on CD-ROMs. The online focus of SAO is predominantly on the communications components of the program, and student answers to these survey questions suggest that that focus is extremely effective.

Making effective use of online discussion forums has its challenges for university instructors who are used to the immediacy and 'just in time' nature of face-to-face teaching. The reliability and versatility of the online technology is always an issue, and both instructors and students need expert support. Discussion forums may well work better for some age groups and subgroups than for others, though, for example, this study has found no significant difference in SAO for either the participation rate or the assessment results for female versus male students in discussion forums. It is planned to carry out follow up studies to probe the way female and male students interact in discussion forums more generally, and also to investigate the participation of different age groups and non-native English speakers in SAO.

SAO is a fully online program predominantly made up of articulate, 'mature age' astronomy enthusiasts, who mostly never meet but relish the opportunity to interact via discussion forums. However the use of asynchronous discussion forums is becoming more prevalent 
as a supplement to face-to-face instruction with predominantly younger student groups at many universities. A proposed extension of this study involves exploring the use of discussion forums for young undergraduates attending on-campus classes (although not in astronomy). Anecdotal experience suggests that forums can be a significant aid to learning in on-campus teaching too, especially where face-to-face tutorial support is limited. Introductory astronomy is a discipline where the development of clear conceptual understanding is crucial: it can be argued that even a mathematically oriented course in astrophysics will be ineffective if students do not first possess a clear understanding of fundamental astronomy concepts. Experience with SAO suggests that in-depth discussions between students are a powerful tool for the development of conceptual understanding, and that asynchronous, online discussion forums can be an effective way to conduct these discussions. However this is anecdotal evidence which requires further study, both in SAO and in situations where discussion forums are used in other teaching contexts. Swinburne University of Technology does not conduct undergraduate astronomy classes, however it would be very interesting to see studies by others on the use of astronomy discussion forums with on-campus undergraduates.

\section{References}

Germany, L., \& Mazzolini, M. 2002, in Proceedings of the Tenth United Nations/European Space Agency Workshop on Basic Space Science: Exploring the Universe - Sky Surveys, Space Exploration, and Space Technologies, Seminars of the United Nations Program on Space Applications, ed. Hans J. Haubold \& S. D. D. Rughooputh (New York: United Nations), 13

Mazzolini, M. 2000, PASA, 17, 141

Mazzolini, M., \& Maddison, S. 2002, Computers \& Education, submitted

Paloff, R. M., \& Pratt, K. 1999, Building Learning Communities in Cyberspace - Active Strategies in the Online Classroom (San Francisco: Jossey-Bass)

Paloff, R. M., \& Pratt, K. 2001, Lessons from the Cyberspace Classroom - the Realities of Online Teaching (San Francisco: Jossey-Bass) 